\title{
Varicella-zoster virus infection in pregnancy
}

\author{
Geta Vancea1,2, Petre I. Calistru ${ }^{1,2}$, Emanoil Ceausu ${ }^{1,2}$ \\ 1"Dr. Victor Babes" Infectious and Tropical Diseases Clinical Hospital, Bucharest, Romania \\ 2"Carol Davila" University of Medicine and Pharmacy, Bucharest, Romania
}

\begin{abstract}
Varicella-zoster virus infection (VVZ) is an important cause of morbidity, with global spread, which causes two distinct clinical forms of disease: varicella and shingles. Primary infection with VVZ during pregnancy can have important consequences for both maternal and fetal health. Epidemiological data shows that vaccination significantly reduced the number of VVZ infections in both mothers and fetuses. Vaccination strategies vary according to the individual at risk (maternal, fetal, newborn, child), the moment with the highest infection risk (first-trimester of pregnancy, last trimester of pregnancy or postnatal) and the duration of the protective immunity after vaccination.

The most common complication of chickenpox during pregnancy is varicella pneumonia that is manifested through cough, dyspnea, fever and tachypnoea. The effects of VVZ on the fetus can be: congenital varicella syndrome, premature or spontaneous abortion, neonatal varicella.

Congenital varicella was first described in 1947. Spontaneous abortion rates, premature births and intrauterine death are not higher in pregnant mothers with chickenpox compared to those not infected. Neonatal infection results from the transmission of VVZ from mother to fetus during the perinatal period.
\end{abstract}

Keywords: varicella, herpes zoster, pregnancy, congenital, prophylaxis

\section{INTRODUCTION}

The infection with varicella-zoster virus (VVZ) determines two distinct clinical forms of the disease: varicella and shingles. Varicella, the primary infection with VVZ, results in generalized vesicular rash and the recurrence of infection, herpes zoster, causes a localized disease.

Varicella is responsible for 4.2 million hospitalizations and 4,200 deaths each year, worldwide, being a highly contagious disease that can be prevented by vaccination (1). In the countries where vaccination was introduced the incidence of cases, hospitalizations and deaths fell substantially $(2,3)$.

During pregnancy the infection can be transmitted to the fetus and, depending on the moment of the infection, it may cause congenital varicella, neonatal varicella or herpes zoster in the first part of childhood. If varicella occurs between 8-20 weeks the fetus presents the risk of congenital chickenpox syndrome. If the mother acquires varicella immediately before or after birth, the child is at risk of developing neonatal chickenpox.

Maternal varicella during pregnancy is also associated with the development of shingles in childhood (4).

Primary infection with VVZ during pregnancy may have significant consequences for both maternal and fetal health. The appearance of herpes zoster in pregnancy is not associated with a significant risk of congenital malformations.

Pregnant women, unborn babies and newborns represent three high-risk populations that can be protected against chickenpox by vaccination. There are some specific protocols regarding maternal immunization (5). Vaccination strategies vary according to the individual at risk (maternal, fetal, newborn, child), the moment with the highest infection 
risk (first-trimester of pregnancy, last trimester of pregnancy or post-natal) and the duration of the protective immunity after vaccination (5).

Although the incidence of chickenpox in adults aged over 20 is below $2 \%$, one quarter of all deaths from VVZ infection are recorded in this age group (6). Pregnant women who fall into this age group are also exposed to a substantial risk of morbidity and mortality.

\section{ETIOLOGY}

Varicella-zoster virus is a DNA virus from the order of of Herpesvirales, the Herpesviridae family, the Alphaherpesvirinae subfamily, Varicellovirus genus (7). It is exclusive to humans, with a diameter of approximately $175 \mathrm{~nm}(8,9)$.

With a linear double-stranded DNA genome of approximately 125,000 base pairs and 71 ORFs genes, it is the smallest of human herpes viruses, being the first to be completely sequenced (10). It presents about 40 genes similar to other herpes human viruses (11).

The viral genome is located in an icosahedral capsule (162 capsomeres), which is surrounded by a protein layer and encapsulated in a tire of polyamines, lipids and glycoproteins. The enveloped glycoproteins facilitate penetration of the virus into cells (12). There is only one VVZ serotype with at least 9 clades, which varies depending on the geographical area. Most viruses in the Western Hemisphere belong to the clades 1 and 3; clade 2 is commonly found in Asia (13).

\section{EPIDEMIOLOGY}

Chickenpox is present all over the world. It has a distinct seasonal pattern in temperate areas where the highest incidence is in winter and spring (14). Prior to the introduction of vaccination the annual incidence of varicella in many European countries was appropriate for the newborn cohort (15).

Epidemiology is different in tropical countries where there is no obvious seasonality. Infection is less commonly acquired in infancy in tropical areas compared to temperate countries, so only $25-85 \%$ of the subjects have primary infection before the age of 15 (14). Different hypotheses have tried to explain this difference, such as: viral inactivation due to high ambient temperatures, race, interference from other viruses and lack of exposure due to tropical living conditions, where VVZ does not circulate very much (14).

Epidemiology of the infection has changed in industrialized countries due to increased immigration and/or increased coverage by vaccination; the incidence of infection decreased by $57-95 \%$ in all age groups where vaccination is widespread $(14,15)$.

The average incidence of chickenpox in pregnancy is estimated at 0.7-3 cases per 1,000 pregnancies $(16,17)$.

Epidemiological data shows that vaccination reduced the number of VVZ infections in both mothers and fetuses. In Australia, between 2006 and 2009, the incidence of congenital chickenpox reached 0.19 per 100,000 live births per year and the incidence of neonatal infections was only 2 per 100,000 live births (18). An 85\% decrease of chickenpox cases in newborns in the postvaccinal period compared to the prevaccinal one has been registered.

\section{Source of infection}

The human being is the only reservoir known by VVZ, the source of infection being the chikenpox or herpes zoster patient. There are no healthy carriers of VVZ.

\section{Transmission}

The transmission of chickenpox is direct from person to person through infected respiratory secretions from the nasopharyngeal mucosa (aerosols, Flugge drops) and penetrates through the conjunctival, nasal and oral mucosa. Transmission is also accomplished by direct contact with the virus content of the skin vesicles or with freshly contaminated objects with the respective vesicular fluid. Susceptible individuals may also get infection from shingles, but transmission rates are considerably lower and require direct contact with skin lesions.

Vertical transmission from mother to child is rare. This can be done in uterus, perinatal or postnatal. Intrauterine or perinatal infection of the fetus is facilitated by transplacental transport, while postnatal varicella is transmitted by respiratory droplets or direct contact with a pacient with varicella (19). 
Transmission of the varicella zoster virus to the fetus during the herpes zoster episode is rare $(4,20)$. Low transmission rates may be associated with the preexistence of maternal VVZ antibodies and lower levels of viremia that accompany reactivation of VVZ infection.

The receptivity is total in seronegative individuals for VVZ. Varicella is highly transmissible with secondary attack rates in people who are $90 \%$ susceptible to pre-vaccination (6).

\section{Contagiousness}

Patients are infectious 1-2 days prior to the occurrence of the chickenpox eruption until the lesions are in crusts.

\section{PATHOGENESIS}

After transmission to a susceptible host, VVZ proliferates in the oropharynx, infects $\mathrm{T}$ cells and produces a first viremia; $T$ cells transport the virus into the skin and other organs. The infection is initially controlled by innate immunity that slows viral multiplication at the epidermal level and thus allows adaptive immunity to develop (21).

Replication of the virus at epidermal levels with overcoming local antiviral responses results in the characteristic lesions of varicella. The incubation period ranges from 10 to 21 days.

Viral replication is controlled by both the innate and the adaptive immune responses, with initially mediated defense by NK cells and type 1 interferons (11).

VVZ-specific T cells, which are essential to stop the viremic phase and to allow varicella healing, become detectable 1-3 days after the rash occurs. These VVZ-specific T-cells target antigens that include glycoprotein $\mathrm{gE}$ of VVZ, protein 62 and other viral proteins (21). The severity of the infection is inversely correlated with the rapidity of $\mathrm{T}$ cell proliferation and the intensity of this response.

People with T-cell deficiency, such as patients with haematological malignancies affection, in chemotherapy or with HIV, usually have severe chickenpox (22). VVZ-specific T-cell immunity is initiated by a response of type 1 helper $\mathrm{T}$ cells (Th1), which produce characteristic cytokines such as interleukin (IL) -2, IL-12, tumor necrosis factor (TNF)-a and IFN -gamma.
$\operatorname{IgM}, \operatorname{IgA}$ and $\operatorname{IgG}$ antibodies in comparison with VVZ proteins are detectable within the first 3 days of the appearance of the rash, but early antibody production is not associated with reducing the clinical severity of the disease (21). After curing the initial infection, VVZ remains latent in the neurons of the cranial nerve, of the spinal dorsal sensitive roots, enteric and autonomic ganglia where it persists throughout life. During latency, VVZ DNA is circular, nonreplicative, with a very narrow pattern of expression of viral genes (21). The mechanism by which VVZ is transferred from infected lymphocytes to neurons and the mechanism by which neurons remain latently infected is unknown (14).

Recent evidence suggests that the latency of both VVZ and herpes simplex virus type 1 is regulated epigenetically (21).

T-cells with specific VVZ memory, with a mixed central phenotype and effector are important for maintaining VVZ latency, immunity being periodically stimulated by endogenous (subclinical) reactivation and exogenous reexposure to VVZ (22).

The latent virus retains the ability to resume replication and cause recurrent clinical disease. The increase of VVZ reactivation with age is largely attributable to the decrease in $\mathrm{T}$ cells and less to humoral immunity (21). However, it is unclear whether this effect is due to the reduction in the quantity or change in the quality of circulating $\mathrm{T}$ cells.

As with varicella, the mediated immune response of T-cell specific in herpes zoster is inversely correlated with the severity of the disease and with the risk of serious consequences, such as postzoster neuralgia (22).

Primary infection with VVZ generally results in lifetime immunity; reports of reinfection cases are rare.

\section{Maternal infection}

Primary infection in the mother causes viral replication in the regional tonsils and lymph nodes and possibly in salivary glandular duct tissue, lasting 4-6 days, with a first viremia leading to spreading to other internal organs where replication continues. Later VVZ is again released into the blood (secondary viremia) and invades skin tissue resulting in the exanthemus in about 14 to 21 days. 
The incubation period in the mother is thus characterized by two phases of viremia, between day 4 and 6 and, respectively, between 10th and 14th day after exposure. The second viremia seems more important and the risk of transplacental passage of the virus is greater.

Anti-VVZ antibodies appear 10-20 days after infection and are transmitted to the fetus, providing passive immunity. VVZ has an important neurotropic affinity so its effects on the nervous system can explain many malformations.

Most reported cases refer to an infection that occurred prior to 20 weeks of gestation; beyond this term the occurrence of congenital varicella is exceptional. After 20 weeks of pregnancy the mechanism is different and determines the neonatal chickenpox (16).

\section{Intrauterine infection}

The mechanism of VVZ infection in the uterus is incompletely known. It is accepted that maternal viremia leads to placenta infection and subsequently to fetal infection. VVZ DNA was detected in several fetal organs and histological examination of the placenta revealed granulomas and acute inflammation $(2,3)$. VVZ fetal replication sites are not known. It has been suggested that the fetus develops varicella in utero followed by healing, with subsequent infection of the dorsal root ganglia. This results in the destruction of the cells of the nervous tissue, with the appearance of changes in the fetal limbs observed in the congenital varicella.

\section{CLINICAL FEATURES OF MATERNAL INFECTION}

\section{Uncomplicated varicella}

Uncomplicated forms of chickenpox present characteristic, pruriginous and generalized rash, occurring in successive waves, with a prodrome of fever, asthenia, myalgia. Skin lesions characteristically evolve from poppy, papules, blisters with clear content, then cloudy content, with the formation of crusts that temporarily leave a hypopigmented area after detachment.

\section{Complicated varicella}

The most common complications of varicella occur mainly in adults and are meningitis, encepha- litis, cerebellar ataxia, pneumonia, myocarditis, glomerulonephritis, death. The most common complication of chickenpox during pregnancy is varicella pneumonia that is manifested through cough, dyspnea, fever and tachypnoea. Pneumonia usually develops during the first week of rash eruption. Clinical evolution is unpredictable, with rapid progression with hypoxia and respiratory failure (24). Radiologically there is evidence of diffuse, milliar or nodular infiltration, with peribronchial distribution affecting both lungs. Varicella pneumonia complicates $10-20 \%$ of cases of varicella in pregnancy.

Pregnant women with VVZ pneumonia should be hospitalized for monitoring and initiating antiviral therapy; up to $40 \%$ of them may require mechanical ventilation. The risk for pneumonia increases with increasing gestational age, due to the maternal immunosuppression and a purely mechanical factor of diaphragm ascension due to the increase in the pregnant uterus (25).

Mortality in severe cases requiring mechanical ventilation has fallen from $45 \%$ to $3-14 \%$ at present (25).

\section{Diagnostic}

Diagnosis of varicella in pregnancy is usually a clinical diagnosis. If there are doubts about clinical diagnosis, VVZ infection can be confirmed quickly by detecting viral DNA by PCR from the product obtained by blistering the vesicle base or by detecting VVZ antigen by immunofluorescence. VVZ can be cultivated from vesicular fluid, but the virus replicates slowly, so cultures are much less used than direct detection techniques.

Serological testing is not necessary for the diagnosis of maternal chickenpox.

Fetal effects of VVZ infection can be: congenital varicella syndrome, premature or spontaneous abortion, neonatal varicella.

\section{CONGENITAL VARICELLA}

Congenital varicella was first described in 1947. It is associated with a $30 \%$ mortality rate in the first months of life and a $15 \%$ risk of developing herpes zoster between the 2nd and the 41th month of life. However, despite the unfavorable prognosis, there is also long term survival (25). 


\section{The incidence of congenital varicella depending on the age of gestation}

Varicella, in the first two trimesters of pregnancy, results in an intrauterine infection of up to $25 \%$ of cases, but congenital abnormalities only occur in just $12 \%$ of infected fetuses (25). The maternal chickenpox occurring in the first 20 weeks of pregnancy has been associated with an incidence of congenital chickenpox of $0.91 \%$ (26). Seldom cases of congenital varicella and maternal varicella were reported from 20 to 28 weeks of gestation, but no such cases were recorded after 28 weeks gestation (26).

Although the clinical characteristics of congenital varicella are multi-system, some tissues and organs are selectively affected $(27,28)$ :

- cutaneous scars in a dermatomal pattern

- limb hypoplasia

- neurological abnormalities (mental retardation, microcephaly, hydrocephalus, seizures, Horner's syndrome)

- ocular anomalies (optic atrophy, cataracts, corioretinitis, microftalmia, nystagmus)

- limb abnormalities (hypoplasia, atrophy, paresis)

- gastrointestinal abnormalities (gastroesophageal reflux, atresia or intestinal stenosis)

- low birth weight

If skin lesions occur in approximately $70 \%$ of cases, limb hypoplasia was recorded in $46-72 \%$ of cases. Neurological abnormalities have a frequency of up to $62 \%$ of cases and eye disorders have been identified up to $52 \%$ (25). Muscle hypoplasia, developmental delay, gastrointestinal, genito-urinary tract abnormalities and cardiovascular system occur in $7-24 \%$ of cases. Bone hypoplasia and skin lesions have dermatomeric location (17).

\section{The mechanism of producing congenital varicella}

The occurrence of this condition is most likely due to $\mathrm{VVZ}$ reactivation in utero, a situation similar to that of shingles. The short period of latency between primary infection and reactivation can be determined by the immaturity of mediated fetal immunity (31). The evidence for reactivation come from the dermatomeric appearance of skin lesions, similar to herpes zoster, from segmental changes in the development of the musculoskeletal system and system dysfunction of the somatic and autonomic nervous systems (24).

\section{Diagnosis of congenital varicella}

\section{Prenatal diagnosis}

Following maternal infection the risk of congenital varicella can be estimated by testing fetal blood or amniotic fluid by PCR for VVZ-DNA along with ultrasonography for fetal anomaly detection (27).

PCR testing for VVZ-DNA is performed between weeks $17-21$ of gestation. A detailed ultrasonographic anatomical assessment will be done no sooner than five weeks after maternal infection to highlight anomalies (17).

The normal results of ultrasonography and laboratory tests suggest a low risk of congenital varicella. A normal ultrasound with detectable DVVZDNA suggests a potential risk and a resumption of ultrasound at 22-24 weeks is indicated. If repeated echography is normal the risk of congenital varicella is low. If ultrasonography shows evidence of pregnant congenital varicella the pregnant mother must be notified about the probable fetus disease (17).

Serological testing of the fetus has a limited utility due to its low sensitivity and specificity.

\section{Postnatal diagnosis}

Diagnosis of congenital varicella requires the following criteria (25):

- history of maternal infection with varicella in the first or second trimester of pregnancy

- the presence of fetal anomalies compatible with congenital chicken pox syndrome

- evidence of intrauterine infection with VVZ.

Evidence of intrauterine infection may include any of the following:

- VVZ-DNA detection in the newborn

- the presence of VVZ-specific IgM antibodies in umbilical cord blood

- persistence of VVZ-specific IgG after the age of seven months

- the appearance of herpes zoster in the first year of life.

Diagnosis of congenital varicella can be confirmed by the presence of maternal varicella in pregnancy along with cutaneous dermatomeric lesions, with or without neurological signs, ocular defects, limb malformations and neonatal convulsions. Retrospective evidence of maternal IgG- 
VVZ seroconversion during pregnancy may be useful.

Almost $20 \%$ of infants with intrauterine VVZ infection develop neonatal or infantile zoster herpes, which is usually without complications $(25,29)$.

Serum fetal detection of specific $\operatorname{IgM}$ is useful in confirming intrauterine infection, but serology has a low sensitivity.

\section{Maternal zoster and congenital varicella}

The particularity of VVZ infection remaining latent in the sensitive spinal nodes, corroborated with uterine innervation which is ensured by the T10-L4 spinal nerves could, theoretically, cause the intrauterine infection with this virus. However, cases of congenital varicella have not been reported following the onset of herpes in the first two trimesters of pregnancy and no clinical or serological evidence of VVZ infection has been reported in infants whose mothers have developed perinatal herpes zoster (25).

Newborns with congenital varicella do not have an active disease, but only sequelae.

\section{PREMATURITY AND SPONTANEOUS ABORTION}

Varicella is not associated with first-trimester abortion (25). Spontaneous abortion rates, premature births and intrauterine death are not higher in pregnant mothers with chickenpox compared to those not infected.

\section{NEONATAL VARICELLA}

Neonatal infection results from the transmission of VVZ from mother to fetus during the perinatal period.

Neonatal chickenpox may occur by transplacental transmission, ascending infection or by the neonatal respiratory tract. Neonatal chickenpox occurring in the first 10-12 days of life is caused by intrauterine transmission, whereas after this interval it is due to postnatal infection of the newborn.

When maternal varicella occurs 1-4 weeks before birth, up to $50 \%$ of newborns will be infected and $23 \%$ of them will develop chickenpox.

The clinical picture of neonatal varices is variable, ranging from mean to severely disseminated infection. Newborns from chickenpox infected mothers who appeared five days before and two days after birth had the highest risk of severe illness and unfavorable development. The newborn will experience a disseminated infection with haemorrhagic rash, pulmonary, hepatic, meningeal, severe prognosis (16).

Increased risk of severe illness also presents babies under 28 weeks of gestation or weighing less than $1,000 \mathrm{~g}$ who do not have active maternal $\mathrm{IgG}$ antibody transfer in the last trimester of pregnancy and the child's immune mediated immune response is not sufficient to prevent the hematogenous spread of VVZ (16).

In the period before neonatal intensive care use of immunoglobulins and antivirals, the mortality rate in neonatal varicella was $31 \%$, but today it decreased to $7 \%$.

Diagnosis of neonatal chickenpox is essentially clinical on the presence of generalized, vesicular, polymorphic rash in a newborn from a mother exposed to VVZ infection or with chronic varicella symptoms during the perinatal period.

In uncertain cases the diagnosis is confirmed by PCR, a high specificity and sensitivity test that detects VVZ from vesicle fluid, vesicle scarring, crust, tissue biopsy, LCR. It also allows differentiation between wild and vaccine strains.

Serological tests are useful in diagnosis but require acute serum also from convalescence.

In neonatal infection, these sera have an elevated IgG VVZ titer, as opposed to uninfected newborns with low antibody titers from passive transfer from the mother. IgM antibodies reflect a recent infection but can cross-react with herpes simplex virus.

\section{MANAGEMENT OF MATERNAL INFECTION}

\section{Varicella treatment in pregnancy}

Varicella is treated with oral aciclovir $(800 \mathrm{mg}$ five times a day, 7 days) for all pregnant women with uncomplicated disease. Initiation of treatment within the first 24 hours of onset of the disease is associated with a faster healing of cutaneous lesions and shorter duration of fever (30).

Pregnancy varicella pneumonia is a medical emergency and requires treatment with intravenous aciclovir (10 mg/kg every eight hours). The risk- 
benefit ratio of maternal varicella treatment with aciclovir exceeds any theoretical concern about fetal toxicity; no specific pattern of congenital malformations was attributed to acyclovir administered in pregnancy (31). Although acyclovir crosses the placenta, it has not been shown that this antiviral agent decreases the risk of congenital chickenpox syndrome (32).

Treatment of herpes zoster during pregnancy is similar to that of non-pregnant adults $(800 \mathrm{mg} 5$ times a day). There is no evidence that pregnant women with herpes zoster have a higher risk of complications and it is recommended to treat only those with more than 50 skin lesions and acute neuritis (33).

\section{TREATMENT OF VARICELLA IN NEWBORN}

Newborns with severe varicella, with pneumonia, encephalitis, hepatitis or thrombocytopenia are treated with intravenous acyclovir, $30 \mathrm{mg} / \mathrm{kg} /$ day, in 3 divided doses for 10 days $(34,35)$. Acyclovir is not teratogenic, crosses the placental barrier, but fetal serum concentrations only reach $15-20 \%$ of maternal concentrations. Treatment of neonatal varicella has eliminated mortality and significantly reduced sequelae. This treatment should be initiated as soon as possible since onset of viral replication stops 72 hours after the onset of the eruption.

The use of aspirin should be avoided because of the increased risk of developing Reye syndrome.

\section{Breastfeeding}

The excretion of varicella-zoster virus in breast milk has been demonstrated by detecting VZV DNA at this level (36). Breastfeeding is encouraged in newborns exposed to or infected with varicella because antibody in breast milk may be protective. Breastfeeding is recommended regardless of the mother's immune status (16).

\section{PROPHYLAXY}

\section{Pre-exposure prophylaxis}

Primary varicella prophylaxis is achieved by vaccination. The vaccine (vOKA) was developed in Japan in 1974 to lower the fatal complications of varicella in immunodeprimed children (10). There are currently 3 commercial vaccine varieties for the prevention of varicella and one for the prevention of shingles.

Vaccination with live attenuated vaccine (Varilrix, Varivax) is indicated for women of childbearing age and no history of clinical varicella. The vaccination schedule is based on the administration of two doses at intervals of 4 to 8 weeks or between 6 and 10 weeks depending on the vaccine used. Contraception is recommended in the first month after vaccination.

Vaccination is contraindicated in pregnant women because of the theoretical risk of vertebral transmission of the vaccine. Vaccination is possible postpartum, including during lactation. Vaccine virus has been shown do not pass into breast milk (17). Post-exposure vaccination administered in the first 3 to 5 days reduced the incidence of varicella by $90 \%$ in exposed subjects.

\section{Post-exposure prophylaxis}

Prevention is targeted at susceptible hosts without a history of infection or prior serological evidence. Assessment of susceptibility is performed anamnestically and by serological determinations prior to immunoprophylaxis.

Passive immunization of the newborn may alter the clinical development of neonatal chickenpox, but does not prevent the disease and, although low, the risk of death is not eliminated (38). Therefore, aciclovir treatment should be promptly administered at a dose of $10 \mathrm{mg} / \mathrm{kg}$ every 8 hours intravenously for 7 days in case of suspicion of neonatal varicella.

To reduce mortality in neonatal varicella the date of birth can be delayed, allowing maternal antibodies to pass through the placental barrier (23). No neonatal chickenpox therapy is indicated if the onset of maternal eruption is more than 7 days prior to birth.

Post-exposure immunoprophylaxis for the prevention of maternal varicella is achieved by passive immunization with intramuscular varicella-specific immunoglobulins. The recommended dose is 125 $\mathrm{u} / 10 \mathrm{~kg}$ body to a maximum of $625 \mathrm{u}$. In the absence of these specific immunoglobulins intravenous immunoglobulins at a dose of $400 \mathrm{mg} / \mathrm{kg}$ body weight may be used.

Passive immunization can reduce viremia and reduce the risk of mother-to-child transmission. 
The management of newborn exposure to varicella involves both post-exposure prophylaxis and isolation. The specific intervention depends upon the timing of exposure, the mother's serologic status and gestational age. Immunoglobulins specific for varicella-zoster are purified human immunoglobulins obtained from plasma containing high levels of anti-varicella antibodies.

Immunoprophylaxis after exposure to $\mathrm{VVZ}$ (varicella or herpes zoster) is recommended for patients at increased risk for severe complications and who cannot receive a VVZ vaccine $(26,38,39)$ :

- neonates whose mothers have signs and symptoms of varicella around the time of delivery (within five days before or two days after)

- hospitalized premature infants born at $\geq 28$ weeks of gestation whose mothers do not have documented immunization, serologic immunity or prior documented history of varicella infection

- hospitalized premature infants born at $<28$ weeks of gestation or who weigh $<1,000$ grams at birth regardless of maternal history of varicella or vaccination.
- pregnant women who do not have immunity to VVZ.

Passive immunization after exposure to chickenpox can be done up to 10 days.

The recommended dose is 125 units, intramuscularly, for newborns weighing over $2.1 \mathrm{~kg}$ and 62.5 units for those under $2 \mathrm{~kg}$. If specific immunoglobulins are not available intravenous immunoglobulins or prophylaxis with acyclovir can be given.

Isolation for the mother and infant depends upon whether there is active disease or the timing of exposure. Thus, the mother with varicella is isolated and the newborn will be isolated from the mother until she is no longer contagious. Any newborn who presents chickenpox in the hospital will be isolated.

If the mother presents varicella 21 days before delivery she will no longer be isolated, but the newborn will be isolated from other infants. A seronegative mother exposed to varicella 6-21 days prior to delivery should be isolated from other patients because she may develop varicella while hospitalized.

\section{REFERENCES}

1. WHO. Varicella and herpes zoster vaccines: WHO position paper. Wkly Epidemiol Rec 2014;89:265-88

2. Leung J, Harpaz R. Impact of the maturing varicella vaccination program on varicella and related outcomes in the United States: 1994-2012. J Ped Infect Dis Soc 2016; 5:395-402

3. Henry O, Brzostek J, Czajka $\mathrm{H}$ et al. One or two doses of live varicella virus-containing vaccines: Efficacy, persistence of immune responses, and safety six years after administration in healthy children during their second year of life Vaccine 2018; 36: 381-387

4. Enders G, Miller E, Cradock-Watson J et al. Consequences of varicella and herpes zoster in pregnancy: prospective study of 1739 cases. Lancet 1994; 343:1548

5. Vermillion MS, Klein SL. Pregnancy and infection: Using disease pathogenesis to inform vaccine strategy. NPJ Vaccines 2018; 3:6

6. Marin M, Güris D, Chaves SS et al. Prevention of varicella: Recommendations of the Advisory Committee on Immunization Practices. (ACIP) MMWR Recomm Rep 2007; 56:1

7. De Paschale M, Clerici P. Microbiology laboratory and the management of mother-child varicella-zoster virus infection. World J Virol. 2016 Aug 12; 5. (3): 97-124

8. Quinlivan M, Breuer J. Molecular studies of Varicella zoster virus. Rev Med Virol. 2006;16:225-250

9. Gabutti G. VZV infection: Epidemiology and prevention. J Prev Med Hyg. 2007:48:65-71

10. Breuer J. Molecular Genetic Insights Into Varicella Zoster Virus. (VZV), the vOka Vaccine Strain, and the Pathogenesis of Latency and Reactivation. The Journal of Infectious Diseases 2018;218. (S2):S75-80

11. Arnold N, Messaoudi I. Herpes zoster and the search for an effective vaccine. Clin Exp Immunol. 2017;187. (1):82-92
12. Cohen JI. The varicella-zoster virus genome. Curr Top Microbiol Immunol. 2010;342:1-14

13. Gershon AA. Is chickenpox so bad, what do we know about immunity to varicella zoster virus, and what does it tell us about the future? J Infect. 2017 Jun; 74. (Suppl 1): S27-S33

14. Gershon AA, Breuer J, Cohen Jl et al. Varicella zoster virus infection. Nat Rev Dis Primers. 2015;1:15016

15. Riera-Montes $M$, Bollaerts $K$, Heininger $U$ et al. Estimation of the burden of varicella in Europe before the introduction of universal childhood immunization. BMC Infect Dis. 2017;17. (1):353.

16. Charlier C, Le Mercier D, Salomon LJ et al. Varicelle, zona et grossesse. La Presse Médicale. 2014;43. (6):665-75

17. Methlouthi J, Mahdhaoui N, Bellalah M, Ayache H, Nouri S, Seboui $\mathrm{H}$. La varicelle perinatale: risques et prise en charge foetale et neonatale. Pan Afr Med J. 2017; 28: 233.

18. Khandaker G, Marshall H, Peadon E et al. Congenital and neonatal varicella: Impact of the national varicella vaccination programme in Australia. Arch Dis Child 2011; 96:453.

19. Enright AM, Prober CG. Herpesviridae infections in newborns: varicella zoster virus, herpes simplex virus, and cytomegalovirus. Pediatr Clin North Am 2004; 51:889.

20. Harger JH, Ernest JM, Thurnau GR et al. Frequency of congenital varicella syndrome in a prospective cohort of 347 pregnant women. Obstet Gynecol 2002; 100:260.

21. Warren-Gash C, Forbes H, Breuer J. Varicella and herpes zoster vaccine development: lessons learned. Expert Rev Vaccines. 2017; 16. (12): 1191-1201

22. Ouwendijk WJ, Laing KJ, Verjans GM et al. T-cell immunity to human alphaherpesviruses. Curr Opin Virol. 2013;3. (4):452-460 
23. Nikkels AF, Delbecque K, Pierard GE et al. Distribution of varicellazoster virus DNA and gene products in tissues of a first-trimester varicella-infected fetus. J Infect Dis 2005; 191:540

24. Gnann JW Jr. Varicella-zoster virus: Atypical presentations and unusual complications. J Infect Dis 2002; 186 Suppl 1:S91.33.

25. Lamont R.F, Sobel,J.D, Carrington D, Mazaki-Tovi S, Kusanovic J,P, Vaisbuch E, Romero R, Varicella Zoster Virus. (Chickenpox) Infection in Pregnancy. BJOG. 2011 Sep; 118. (10): 1155-1162.

26. Tan MP, Koren G. Chickenpox in pregnancy: Revisited. Reprod Toxicol. 2006;21. (4):410-420.

27. Koren G. Congenital varicella syndrome in the third trimester. Lancet 2005; 366:1591.

28. Mazzella M, Arioni C, Bellini C et al. Severe hydrocephalus associated with congenital varicella syndrome. CMAJ 2003; 168:561.

29. Sauerbrei A, Wutzler P. Das fetale Varizellen-syndrome. Monatsschrift Kinderheilkunde. 2003;151:209-213.

30. Wallace MR, Bowler WA, Murray NB et al. Treatment of adult varicella with oral acyclovir. A randomized, placebo-controlled trial. Ann Intern Med 1992; 117:358.

31. Reiff-Eldridge R, Heffner CR, Ephross SA et al. Monitoring pregnancy outcomes after prenatal drug exposure through prospective pregnancy registries: A pharmaceutical company commitment. Am J Obstet Gynecol 2000; 182:159.
32. Pacifici GM. Transfer of antivirals across the human placenta. Early Hum Dev 2005; 81:647.

33. Cohen A, Moschopoulos P, Stiehm RE, Koren G. Congenital varicella syndrome: The evidence for secondary prevention with varicellazoster immune globulin. CMAJ 2011; 183:204.

34. Kesson AM, Grimwood K, Burgess MA et al. Acyclovir for the prevention and treatment of varicella zoster in children, adolescents and pregnancy. J Paediatr Child Health 1996; 32:211.

35. Williams H, Latif A, Morgan J, Ansari BM. Acyclovir in the treatment of neonatal varicella. $J$ Infect 1987; 15:65.

36. Yoshida M, Yamagami N, Tezuka T, Hondo R. Case report: Detection of varicella-zoster virus DNA in maternal breast milk. J Med Virol 1992; 38:108.

37. Bialas K.M, Swamy G.K, Permar S.R. Perinatal cytomegalovirus and varicella zoster virus infections: Epidemiology, prevention, and treatment Clin Perinatol. 2015 Mar; 42. (1): 61.

38. Centers for Disease Control and Prevention. (CDC) Updated recommendations for use of VariZIG - United States, 2013. MMWR Morb Mortal Wkly Rep 2013; 62:574.

39. Zhang HJ, Patenaude V, Abenhaim HA. Maternal outcomes in pregnancies affected by varicella zoster virus infections: populationbased study on 7.7 million pregnancy admissions. J Obstet Gynaecol Res 2015; 41:62. 